\title{
Quantifying self-absorption losses in luminescent solar concentrators
}

\author{
Otmar M. ten Kate, ${ }^{*}$ Koen M. Hooning, and Erik van der Kolk \\ Fundamental Aspects of Materials and Energy, Delft University of Technology, \\ Mekelweg 15, 2629 JB Delft, The Netherlands \\ ${ }^{*}$ Corresponding author: o.m.tenkate@ $@$ tudelft.nl
}

Received 24 February 2014; revised 20 May 2014; accepted 14 July 2014; posted 15 July 2014 (Doc. ID 206713); published 8 August 2014

\begin{abstract}
Analytical equations quantifying self-absorption losses in circular luminescent solar concentrators (LSCs) are presented that can easily be solved numerically by commercial math software packages. With the quantum efficiency, the absorption and emission spectra of a luminescent material, the LSC dimensions, and the refractive index as the only input parameters, the model gives an accurate account of the decrease of LSC efficiency due to self-absorption as a function of LSC radius, thickness, and luminescence quantum efficiency. Results give insight into how many times light is reabsorbed and reemitted, the red shift of the emission spectrum, and on how multiple reabsorptions and reemissions are distributed over the LSC. As an example case the equations were solved for a circular LSC containing a Lumogen F Red 305 dye with $80 \%$ luminescence quantum efficiency, and it follows that for an LSC with a $50 \mathrm{~cm}$ radius the self-absorption reduces the number of photons reaching the LSC edge by a factor of four compared to the case when there would be no self-absorption. The equations can just as well be solved for any material for which the optical properties are known like type I and type II quantum dots. () 2014 Optical Society of America

OCIS codes: $\quad$ (260.3800) Luminescence; (350.6050) Solar energy.

http://dx.doi.org/10.1364/AO.53.005238
\end{abstract}

\section{Introduction}

A luminescent solar concentrator (LSC) reduces solar electricity costs by using a relatively inexpensive plate containing a luminescent material that absorbs solar light that enters the plate via its front surface. The luminescent material subsequently reemits the light at longer wavelengths. The reemitted light is largely trapped within the LSC plate because of its higher refractive index as compared to the surrounding air and is waveguided to the edges of the LSC, where photovoltaic (PV) cells are attached. Since the area of these edges can be more than 100 times smaller than the area of the front surface that collects the solar light, less area for the PV cells is needed [1-3].

$1559-128 \mathrm{X} / 14 / 235238-08 \$ 15.00 / 0$

(C) 2014 Optical Society of America
The quantum efficiency of an LSC depends on many different factors, such as the light in-coupling efficiency, the light harvesting efficiency [4], the luminescence quantum efficiency [5], the light trapping efficiency $[\underline{6}]$, and the waveguide efficiency $[\underline{7}, 8]$. But one of the main factors limiting the overall LSC efficiency is the losses associated with selfabsorption [9]. For many luminescent materials considered for LSC applications, such as some organic dyes and luminescent quantum dots, a certain part of the emission spectrum overlaps with the absorption spectrum. This spectral overlap results in substantial reabsorption of the emitted light before the photons can reach the edges of the LSC where the PV cells are attached. Although reabsorption can again result in reemission, nonunity luminescence quantum efficiency and nonunity light trapping efficiency significantly limit the fraction of light that finally reaches the LSC-PV interface $[4,7,10]$. 
Batchelder and co-workers $[11,12]$ described a way to quantify the self-absorption effects analytically. It was realized that self-absorption leads to different generations of luminescence because each reabsorption leads to reemission. A technique was described to determine the reemission distribution over the LSC given the distribution of photons absorbed within the LSC. The set of equations was solved for the simplified case of a semi-infinite rod. Others such as Olson et al. [13], Sansregret et al. [9], Sholin et al. [14], Sahin and Ilan [15], Wilson and Richards [5], and Kerrouche et al. [16] calculated self-absorption losses using a Monte Carlo simulation or ray-tracing approach. Chatten et al. [17] and Fang et al. [18] used a thermodynamic approach to describe selfabsorption losses in LSCs. Sahin and Ilan [19] presented an analytical approach using a generalization of radiative transport theory. Their equations were solved numerically for a semi-infinite rectangular LSC and compared with Monte Carlo simulations.

In this work, an analytical set of equations is given that describe the self-absorption efficiency in a nonscattering circular LSC. As an example, the equations are solved numerically for a circular LSC plate containing a Lumogen $\mathrm{F}$ Red 305 dye, which is equally distributed over the LSC. The sun light enters the LSC via its front surface and the solar cells are attached as a strip to the edge of the circular plate. The advantage of our analytical approach is that more insights can be obtained in the process, such as insights regarding the self-absorption intensity as a function of the location in the LSC and the edge transmission per generation of emission. The derivation of the equations is split into two parts. In the first part in Section 2, the equations that describe self-absorption in a flat circular LSC without any thickness (a 2D circular LSC) are derived. Then, in the second part in Section 3, the procedure is repeated for a $3 \mathrm{D}$ circular $\mathrm{LS} \overline{\mathrm{C}}$ while taking the thickness of the LSC into account.

\section{Self-Absorption in a 2D Circular LSC}

\section{A. Theory}

The transmission of a light beam through a medium with absorption coefficient $\alpha$ is given by the BeerLambert law:

$$
I(x)=I_{0} \exp (-\alpha x)
$$

Here, $I_{0}$ is the original intensity and $I(x)$ the remaining intensity at the distance $x$ traveled. $I(x)$ can also be interpreted as the remaining light intensity going through a line segment $d x$ located a distance $x$ away from a point light source in cases, where the light was emitted in one direction. In the case of an isotropic, circularly radiated point emission, the expression with $x$ replaced by $s$ corresponds to the light intensity going through a circle with radius $s$, where the source point is located in the center of the circle. The derivative of the equation with respect to $s$ then gives the change in intensity as a function of $s$. The difference in intensity going through a circle with radius $s$ and a circle with radius $s+d s$ then equals $-\alpha I_{0} e^{-\alpha s} d s$. The corresponding absorption per unit area $\gamma$ at a distance $s$ from the emission center is therefore

$$
\gamma=\frac{\alpha I_{0} \exp (-\alpha s)}{2 \pi s} .
$$

Consider a 2D circular LSC with radius $R$, as shown in Fig. 1, and with the initial emission distribution $\sigma_{1}\left(r^{\prime}, \varphi^{\prime}, \lambda\right)$ expressed in power per unit area per wavelength, which is a function of the location $\left(r^{\prime}, \varphi^{\prime}\right)$ in the LSC and the wavelength $\lambda$ of the emitted light. This emission will, as shown by Eq. (2), lead to an absorption $d A$ in the infinitesimally small element $s d \xi d s$ in the LSC of

$$
d A=\frac{\alpha \sigma_{1} \exp (-\alpha s)}{2 \pi} d \xi d s .
$$

Integrating this expression over all absorption positions $(s, \xi)$ on the LSC and subsequently integrating over all emission points $\left(r^{\prime}, \varphi^{\prime}\right)$ yields the total absorption $A_{1}(\lambda)$ in the LSC of all light emitted within the LSC:

$A_{1}(\lambda)=\frac{\alpha(\lambda)}{2 \pi} \int_{0}^{R} \int_{0}^{2 \pi} \int_{s_{\min }}^{s_{\max }} \int_{\xi_{\min }}^{\xi_{\max }} r^{\prime} \sigma_{1} \exp (-\alpha s) \mathrm{d} \xi \mathrm{d} s \mathrm{~d} \varphi^{\prime} \mathrm{d} r^{\prime}$

By applying standard geometric relations the variables $s$ and $\xi$ can be expressed as a function of $r^{\prime}, \varphi^{\prime}, r$,

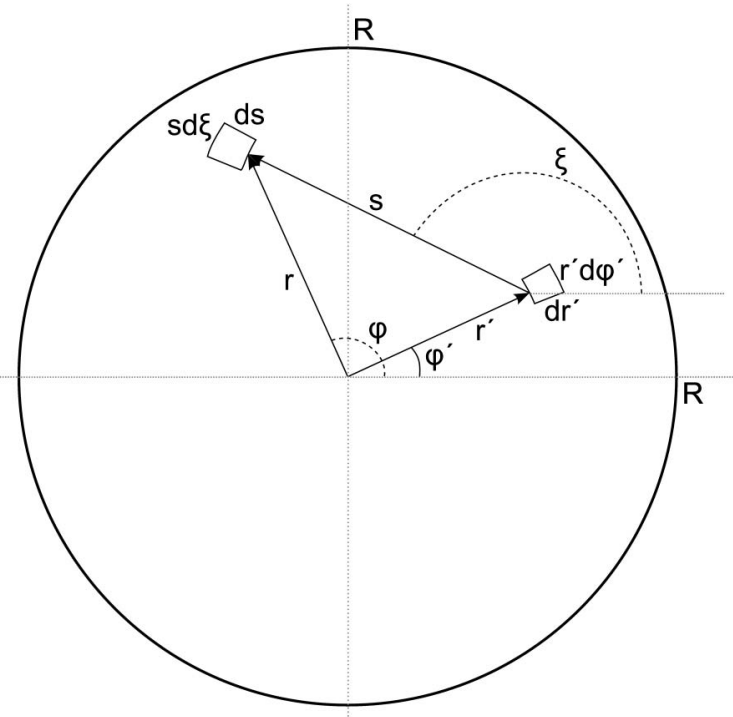

Fig. 1. Schematic representation of light emitted in a 2D circular LSC from a surface element located at position $\left(r^{\prime}, \varphi^{\prime}\right)$ with respect to the center of the circle, which is subsequently reabsorbed in a surface element located at position $(s, \xi)$ with respect to the emission point and located at position $(r, \varphi)$ with respect to the center of the circle. 
and $\varphi$. The integrals over $s$ and $\xi$ can therefore be replaced by integrals over $r$ and $\varphi$ resulting in

$$
A_{1}(\lambda)=\frac{\alpha(\lambda)}{2 \pi} \int_{0}^{R} \int_{0}^{2 \pi} \int_{0}^{R} \int_{0}^{2 \pi}{ }_{-}^{2} r^{\prime} \sigma_{1} \exp (-\alpha s) \mathrm{d} \varphi \mathrm{d} r \mathrm{~d} \varphi^{\prime} \mathrm{d} r^{\prime}
$$

with

$$
s=\sqrt{r^{2}+\left(r^{\prime}\right)^{2}-2 r r^{\prime} \cos \left(\varphi-\varphi^{\prime}\right)} .
$$

Consequently, the reabsorption per unit area $\gamma_{1}(r, \varphi, \lambda)$ at a point $(r, \varphi)$ in the LSC equals

$$
\gamma_{1}(r, \varphi, \lambda)=\frac{\alpha}{2 \pi} \int_{0}^{R} \int_{0}^{2 \pi} \frac{r^{\prime}}{s} \sigma_{1} \exp (-\alpha s) \mathrm{d} \varphi^{\prime} \mathrm{d} r^{\prime} .
$$

The reabsorption per unit area can thus be calculated if the initial emission distribution $\sigma_{1}$ is known. Next, the reemission per unit area $\sigma_{2}$ can be determined given this reabsorption per unit area

$$
\sigma_{2}(r, \varphi, \lambda)=\frac{\Phi_{\mathrm{em}}(\lambda)}{\lambda} \int_{0}^{\infty} \lambda \eta_{\mathrm{LQE}} \gamma_{1}(r, \varphi, \lambda) \mathrm{d} \lambda .
$$

Here, $\eta_{\text {lqe }}$ is the luminescence quantum efficiency and $\Phi_{\mathrm{em}}$ is the normalized photon emission spectrum, which is equal to

$$
\Phi_{\mathrm{em}}=\frac{\sigma_{1}(\lambda)}{\int_{0}^{\infty} \sigma_{1}(\lambda) \mathrm{d} \lambda} .
$$

The so obtained reemission distribution can then be used to determine the next reabsorption $\gamma_{2}$ and so on.

Note that as a result of the fact that a $2 \mathrm{D}$ luminescent plate is considered, any escape cone losses, which are an important loss factor after each emission in any real 3D device, are not included. Therefore, in order to obtain any meaningful results from this $2 \mathrm{D}$ approach, the emission distribution $\sigma$ should be multiplied by a light trapping efficiency $\eta_{\text {trap }}$ in Eq. (7) in order to consider only those photons that are not emitted within the two escape cones. This trapping efficiency depends on the refractive index $n$ of the LSC and is equal to:

$$
\eta_{\text {trap }}=\sqrt{1-\frac{1}{n^{2}}}
$$

All photons that are emitted outside the escape cones and that are not reabsorbed will reach the edges of the LSC, where the solar cells are attached. This edge transmission per generation $i$ is thus given by

$$
T_{i}=\eta_{\text {trap }} \int_{0}^{R} \int_{0}^{2 \pi} \sigma_{i} r^{\prime} \mathrm{d} \varphi^{\prime} \mathrm{d} r^{\prime}-\eta_{\text {trap }} A_{i} .
$$

The total edge transmission per generation is now given by the integral of $T_{i}$ over all wavelengths, and the overall edge transmission is the total sum of all $T_{i}$ over all generations $i$.

Note that the equations mentioned above do not take into account any scattering losses within the LSC. However, the same model might be applied to describe elastic scattering within the LSC by considering scattering as absorption followed by reemission at the same wavelength. In that case, the absorption coefficient $\alpha$ in Eq. (1) should be replaced by a scattering coefficient.

\section{B. Results}

The equations mentioned above can now be used to calculate the self-absorption losses in a $2 \mathrm{D}$ circular LSC. As an example, a polymethyl methacrylate (PMMA) LSC doped with a Lumogen F Red 305 dye with a concentration of $115 \mathrm{ppm}$ is considered with the absorbance and emission intensity as determined by Desmet et al. [20] and shown in Fig. 2. The results per generation for an LSC with a radius of $50 \mathrm{~cm}$ and a refractive index of 1.5 are shown in Table 1 . Although the luminescence quantum efficiency of the Red 305 dye is closer to $100 \%$ [5], an efficiency of $80 \%$ has been used in the calculations in order to show the effect of nonunity luminescence quantum efficiency on self-absorption losses. Hence, that many dyes do not have $100 \%$ luminescence quantum efficiency.

As can be seen from Table 1 , after eight generations of emission and absorption only $0.6 \%$ of the initial amount of absorbed photons is still present in the LSC and can be emitted a 9 th time. The results after eight generations will therefore be close to the results obtained after an infinite number of generations. After these eight generations, $15.2 \%$ of the initial number of absorbed photons has reached the edges of the LSC. In the case where there would be no self-absorption, this would be $59.6 \%$ of the initially absorbed photons. The difference is due to two loss factors. Because of self-absorption, the total losses via the escape cones account for $42.4 \%$ of the initially absorbed photons, as compared to $20.4 \%$ when there would be no self-absorption. The other

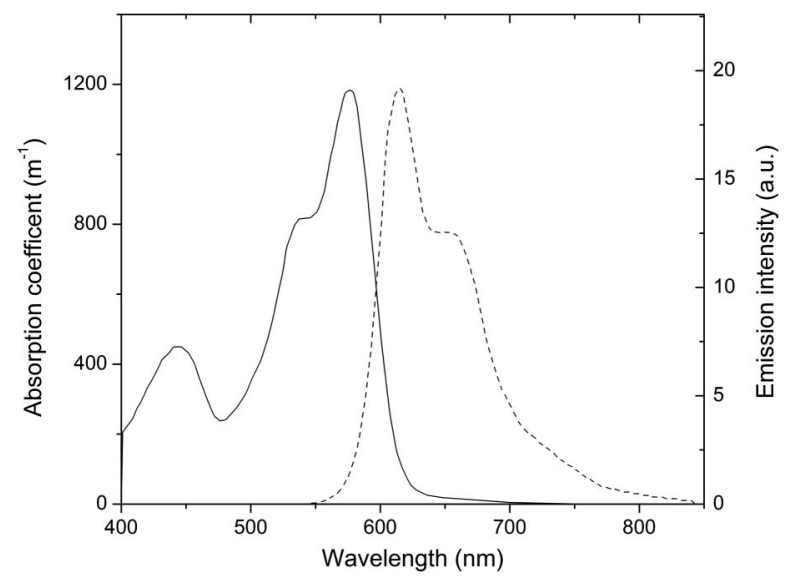

Fig. 2. Absorption coefficient (straight line) and emission spectrum (dashed line) of a PMMA plate doped with 115 ppm Lumogen F Red 305 dye [20]. 


\begin{tabular}{|c|c|c|c|c|}
\hline$i$ & $f_{\mathrm{em}}(\%)$ & $f_{\text {esc }}(\%)$ & $f_{\text {abs }}(\%)$ & $f_{\text {trans }}(\%)$ \\
\hline 1 & 80.0 & 20.4 & 52.3 & 7.3 \\
\hline 2 & 41.8 & 10.6 & 27.3 & 3.9 \\
\hline 3 & 21.8 & 5.6 & 14.3 & 1.9 \\
\hline 4 & 11.4 & 2.9 & 7.5 & 1.0 \\
\hline 5 & 6.0 & 1.5 & 4.0 & 0.5 \\
\hline 6 & 3.2 & 0.8 & 2.1 & 0.3 \\
\hline 7 & 1.7 & 0.4 & 1.1 & 0.2 \\
\hline 8 & 0.9 & 0.2 & 0.6 & 0.1 \\
\hline Total & & 42.4 & & 15.2 \\
\hline
\end{tabular}

loss factor is the nonunity luminescence quantum efficiency, which results in a difference between the number of photons absorbed in generation $i$ and the number of photons emitted in generation $i+1$. These losses account for $41.8 \%$ of the initially absorbed photons, instead of $20 \%$ when there would be no self-absorption.

In Fig. 3, the total transmitted fraction with respect to the initial number of absorbed photons is shown for different luminescence quantum efficiencies as a function of the radius of the 2D LSC. The transmitted fraction decreases by half when the radius increases from 0.1 to $0.5 \mathrm{~m}$. At even larger radii the transmitted fraction further decrease, but less significantly. The reason for this is that the selfabsorption does only have an effect on the emission wavelengths that can be reabsorbed and it does not influence the photons emitted at longer wavelengths. For these photons, the radius is unimportant because the long wavelength photons will reach the LSC-PV edge anyway.

In Fig. 4, the normalized emission spectra of transmitted radiation for LSCs with different radii are shown together with the original emission spectrum

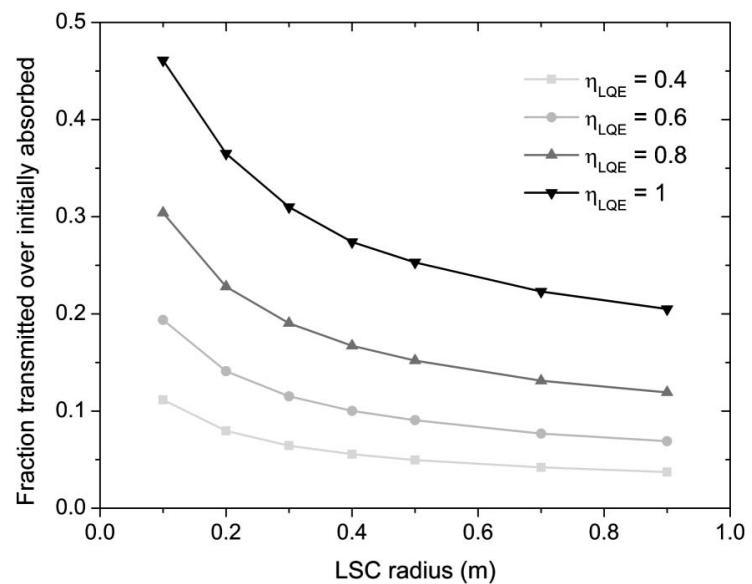

Fig. 3. Fraction of the number of photons transmitted to the LSC-PV edge with respect to the initial number of absorbed photons as a function of the LSC radius for a $2 \mathrm{D}$ circular LSC with a Red 305 dye for different luminescence quantum efficiencies $\left(\eta_{\mathrm{LQE}}\right)$.

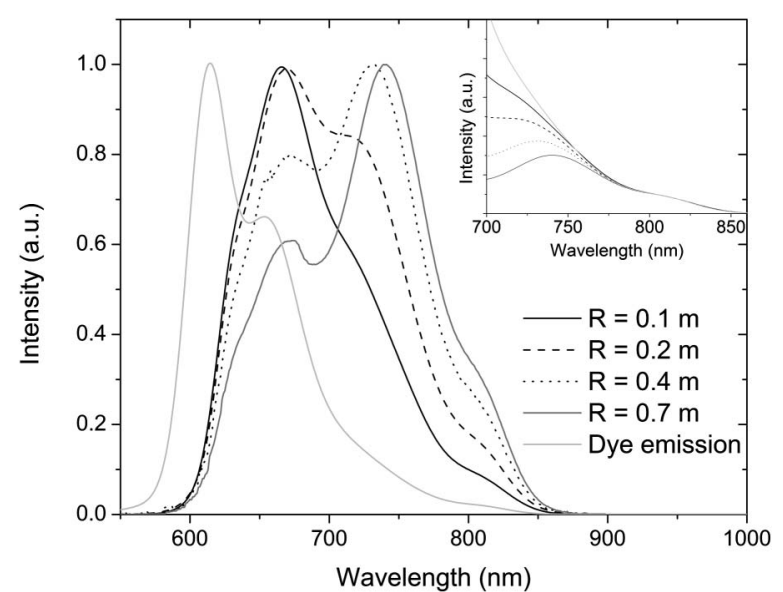

Fig. 4. Dye emission spectrum and edge transmission spectra for 2D circular LSCs with different radii containing a Red 305 dye with $100 \%$ luminescence quantum efficiency. In the main graph, the spectra are normalized on the highest peak, while in the inset the same spectra are normalized on the long wavelength side at $815 \mathrm{~nm}$.

of the dye. The larger the radius of the LSC, the more red-shifted the emission is. The cause of this red-shift is that short wavelength light emitted by the dye is mostly reabsorbed in the LSC, while the longer wavelength light is not reabsorbed. This results in relatively more longer wavelength light that reaches the edges of the LSC as compared to short wavelength light. Larger LSCs have an on average longer photon travel distance, which results in more reabsorption and thus a larger red-shift. The average emission wavelength increases from about $640 \mathrm{~nm}$ for the dye emission spectrum to 680,700 , and $715 \mathrm{~nm}$ for the edge transmission of an LSC with a radius of $0.1,0.2$, and $0.4 \mathrm{~m}$, respectively. Note that the shape of the edge transmission spectra at the long wavelength side is the same as the shape of the emission spectrum of the dye, as is shown in the inset of Fig. 4, since these wavelengths are not absorbed.

\section{Self-Absorption in a 3D Circular LSC}

\section{A. Theory}

Although the 2D model gives nice insights into the processes associated with self-absorption losses, it is a simplification as light emission is assumed to be circular instead of spherical. As a result, the distance a photon needs to travel through the LSC is underestimated with this model. If a photon is emitted under an angle $\theta$ with respect to the line normal to the LSC plain, it actually needs to travel a distance of $\hat{s}=s / \sin (\theta)$ to cover a distance $s$ in the LSC plain, as shown in Fig. 5 .

To account for the extra travel distance in three dimensions, a 3D version of the 2D model from Section 2.A was derived. In this 3D approach the LSC can still be treated as a $2 \mathrm{D}$ plate (in fact a $2 \mathrm{D}$ projection) but now with the path length of the light based on three dimensions. This can be done because the 


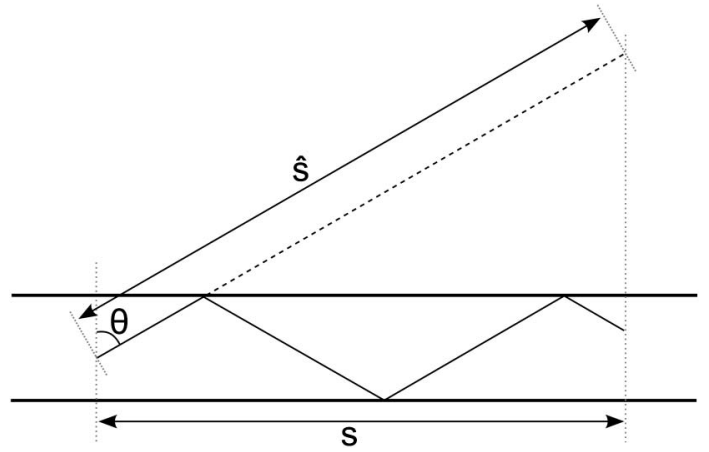

Fig. 5. Directional light transport in a 3D LSC.

distance that a photon needs to travel before it reaches the LSC edge is independent of the depth within the LSC, where the photon is emitted, and also the size of the escape cone, the absorption probability, and the luminescence quantum efficiency are independent of the depth within the LSC.

Comparable to Eq. (2) for the 2D case, the absorption per unit volume $\delta$ at a distance $\hat{s}$ from the emission center (again considered to be an isotropic emission) is given by

$$
\delta=\frac{\alpha I_{0} \exp (-\alpha \hat{s})}{4 \pi \hat{s}^{2}} .
$$

The absorption per unit area $\gamma$ in the LSC plain now corresponds to the integral over all absorptions in the volume elements located in the column that is bounded by the critical angles $\theta_{c}$ and $\pi-\theta_{c}$ and has a cross section $r d r d \varphi$. Comparable to Eq. (5), the total reabsorption for generation $i$ in a 3D circular LSC of all emitted light in the LSC is given by

$$
\begin{aligned}
A_{i}(\lambda)= & \frac{\alpha}{4 \pi} \int_{0}^{R} \int_{0}^{2 \pi} \int_{0}^{R} \int_{0}^{2 \pi} \int_{\theta_{c}}^{\pi-\theta_{c}}{ }_{s}^{r} r^{\prime} \sigma_{i} \\
& \times \exp \left(\frac{-\alpha s}{\sin \theta}\right) \mathrm{d} \theta \mathrm{d} \varphi \mathrm{d} r \mathrm{~d} \varphi^{\prime} \mathrm{d} r^{\prime} .
\end{aligned}
$$

Note that $\sigma_{i}$ in the equation above is the emission per unit area in the LSC plain. It corresponds to the integral over all emissions in a column with height $h$ (the LSC thickness) and cross section $r^{\prime}, d r^{\prime}, d \varphi^{\prime}$. From Eq. (13) it follows that an absorption per unit area for generation $i$ is

$$
\gamma_{i}(\lambda)=\frac{\alpha}{4 \pi} \int_{0}^{R} \int_{0}^{2 \pi} \int_{\theta_{c}}^{\pi-\theta_{c}} \frac{r^{\prime}}{s} \sigma_{i} \exp \left(\frac{-\alpha s}{\sin \theta}\right) \mathrm{d} \theta \mathrm{d} \varphi^{\prime} \mathrm{d} r^{\prime} .
$$

Note that in the 3D approach the trapping efficiency $\eta_{\text {trap }}$ is already accounted for in the reabsorption calculations as the integrals over the $\theta$ range from $\theta_{c}$ to $\pi-\theta_{c}$. Similar to Eq. (11), the edge transmission for a $3 \mathrm{D}$ LSC is given by

$$
T_{i}(\lambda)=\eta_{\operatorname{trap}} \int_{0}^{R} \int_{0}^{2 \pi} \sigma_{i}\left(r^{\prime}, \varphi^{\prime}, \lambda\right) r^{\prime} \mathrm{d} \varphi^{\prime} \mathrm{d} r^{\prime}-A_{i}(\lambda) .
$$

It should be noted that the equations above do not include any thickness for the LSC. The reason for this is that the travel distance of a photon to the LSC edge is independent of the LSC thickness. As a result, the amount of photons reaching the edge does not change with LSC thickness. However, this last statement is not completely true because the escape cone losses slightly depend on the LSC thickness, as will be discussed in Section 3.B.

\section{B. Results}

The results for the $3 \mathrm{D}$ circular $\mathrm{LSC}$ with a radius of $50 \mathrm{~cm}$, which contained the same Red 305 dye as that used for the $2 \mathrm{D}$ case with $80 \%$ luminescence quantum efficiency, are shown in Table 2. The fraction of photons absorbed in each generation is a bit larger as compared to the results for the $2 \mathrm{D}$ model shown in Table 1 . This is due to the on average longer travel distance to the LSC-PV edge in three dimensions, which was not taken into account in the $2 \mathrm{D}$ model. As a consequence, the total transmission over all generations is somewhat smaller in the $3 \mathrm{D}$ model as compared to the 2D model: $14.6 \%$ instead of $15.2 \%$. Note, however, that this difference is small and that the $2 \mathrm{D}$ case thus gives a relatively good approximation.

Note that without self-absorption the transmitted fraction would be $59.6 \%$ if the luminescence quantum efficiency is $80 \%$, which is similar to what was seen for the 2D LSC (Section 2.B). This is because in the absence of self-absorption the amount of photons reaching the edge is independent of the travel distance. Hence, we do not consider scattering or parasitic absorption. This means that for an LSC with a radius of $0.5 \mathrm{~m}$ the efficiency would be reduced by a factor of 4 .

In Fig. 6, the fractions of photons transmitted to the LSC- $\overline{\mathrm{PV}}$ edge are shown as a function of the ra-

\begin{tabular}{|c|c|c|c|c|}
\hline$i$ & $f_{\mathrm{em}}(\%)$ & $f_{\text {esc }}(\%)$ & $f_{\text {abs }}(\%)$ & $f_{\text {trans }}(\%)$ \\
\hline 1 & 80.0 & 20.4 & 52.7 & 6.9 \\
\hline 2 & 42.2 & 10.7 & 27.7 & 3.8 \\
\hline 3 & 22.2 & 5.7 & 14.6 & 1.9 \\
\hline 4 & 11.7 & 3.0 & 7.7 & 1.0 \\
\hline 5 & 6.2 & 1.6 & 4.1 & 0.5 \\
\hline 6 & 3.3 & 0.8 & 2.2 & 0.3 \\
\hline 7 & 1.8 & 0.5 & 1.2 & 0.1 \\
\hline 8 & 1.0 & 0.3 & 0.6 & 0.1 \\
\hline Total & & 43.0 & & 14.6 \\
\hline
\end{tabular}
dius of the 3D LSC for different luminescence quantum efficiencies. The trend is similar to that for the $2 \mathrm{D}$ case shown in Fig. 3, but the transmitted fractions are slightly smaller as explained before. 


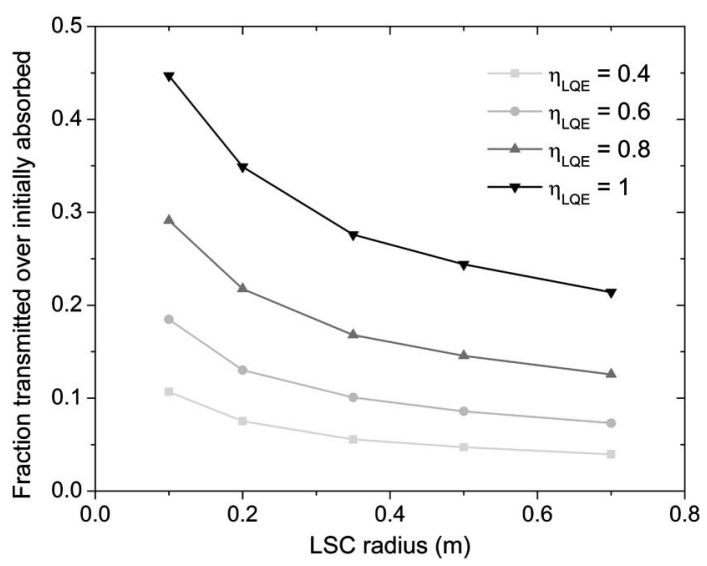

Fig. 6. Fraction of the number of photons transmitted to the LSC-PV edge with respect to the initial number of absorbed photons as a function of the LSC radius for a 3D circular LSC with a Red 305 dye for different luminescence quantum efficiencies.

The initial photon absorption distribution within the LSC and the absorption distribution after three subsequent generations of reemission and reabsorption are shown in Fig. 7 for an LSC with a radius of $0.5 \mathrm{~m}$ and $80 \%$ luminescence quantum efficiency. As can be seen, the number of photons that are absorbed per surface area remains almost constant over the first $40 \mathrm{~cm}$ from the center of the LSC. Only near the edge of the LSC is the absorption density lower. This effect can be explained as follows: the initial emission distribution is constant over the LSC. In that case, all positions more than $10 \mathrm{~cm}$ away from the LSC edge receive the same amount of light, and it is as if the LSC is infinitely large. This is because photons that will be reabsorbed are very likely to be absorbed within $10 \mathrm{~cm}$ from the point of emission, since for most of these photons the absorption coefficient is much larger than $1 \mathrm{~m}^{-1}$. The absorption density is lower only near the edges because these points do not receive light from points beyond the LSC edge.

\section{Cone Angle Absorption}

In the derivation for the self-absorption in a 3D LSC in Section 3.A it has been assumed that all photons emitted within the two escape cones will be lost and that only the photons emitted outside these cones will be trapped. However, this is not entirely true in the case of self-absorption due to cone angle absorption: a photon emitted within the escape cone has a certain chance of being reabsorbed before it can escape from the LSC plate. And since this reabsorption also results in reemission randomly in all directions, a certain fraction of these reemitted photons is trapped and can be attributed to the second generation of emission. As a result, the edge transmission fractions shown in Fig. 6 are slightly underestimated. One can, however, correct for this underestimation by including correction factors for the absorption into the equations.

The chance of a photon being reabsorbed before reaching the LSC surface is a function of the depth $z$ of the emission point within the LSC and the angle of emission $\theta$ with respect to a line perpendicular to the LSC surface. For emission within an LSC with thickness $h$ originating from a point $\left(z^{\prime}, r^{\prime}, \varphi^{\prime}\right)$ with emission intensity per unit volume $\tau_{i}$, the total cone angle absorption $\gamma_{\mathrm{ca}, i}$ due to emission at point $\left(r^{\prime}, \varphi^{\prime}\right)$ in the LSC plain is equal to

$$
\gamma_{\mathrm{ca}, i}\left(r^{\prime}, \varphi^{\prime}, \lambda\right)=\frac{1}{h} \int_{0}^{h} \int_{0}^{\theta_{c}} \tau_{i}\left(r^{\prime}, \varphi^{\prime}, \lambda\right)\left(1-e^{-\alpha z / \cos \theta}\right) \sin \theta \mathrm{d} \theta \mathrm{d} z \text {. }
$$

Note that Eq. (16) is not exactly true for the case in which a photon is emitted within the escape cone close to the edge of the LSC-PV interface. Some of these photons will be able to reach this LSC-PV interface and are thus not absorbed within the LSC. However, due to the very small thickness of the LSC with respect to the radius of the LSC (about a factor of 100 to 1000) these effects account for much less than $1 \%$ of the cone angle absorption and can safely be neglected.

Note that, since the radius of the LSC is much larger than the thickness, the point of emission $\left(r^{\prime}, \varphi^{\prime}\right)$ is very close to the point of absorption $(r, \varphi)$ within the escape cone. As a result, the total cone

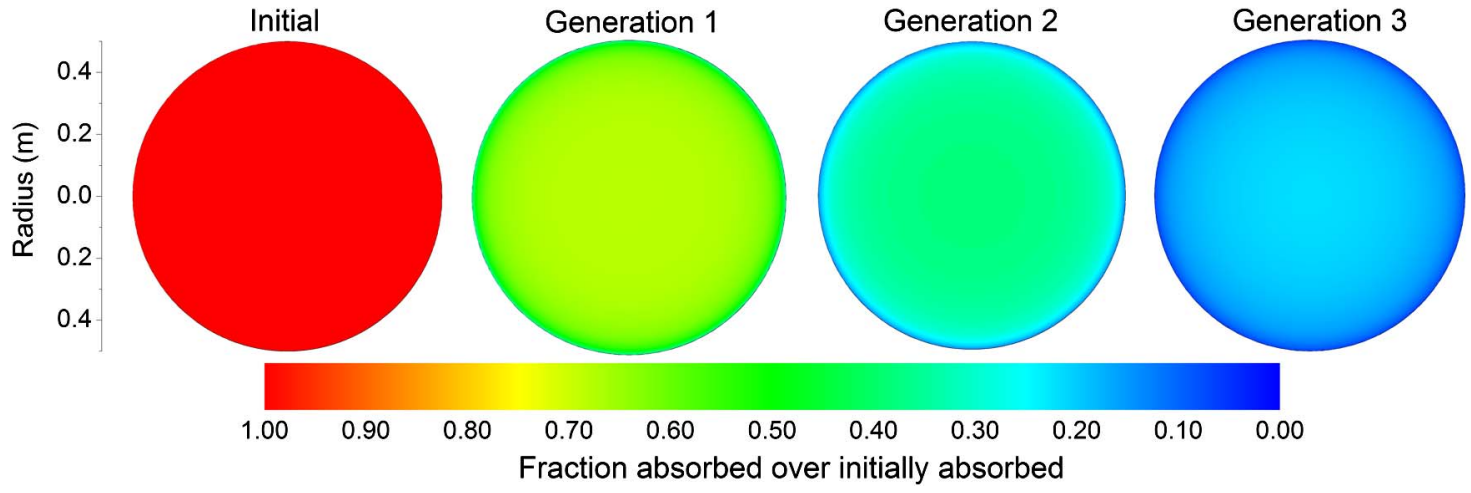

Fig. 7. Distribution of the number of absorbed photons (initial and after the first three generations) with respect to the initial number of absorbed photons as a function of the distance and angle to the LSC center for a 3D circular LSC with a radius of $0.5 \mathrm{~m}$ and $80 \%$ luminescence quantum efficiency. 


\begin{tabular}{|c|c|}
\hline$h(\mathrm{~mm})$ & $f_{\text {trans }}(\%)$ \\
\hline 0.1 & 14.7 \\
\hline 0.5 & 14.9 \\
\hline 1 & 15.2 \\
\hline 3 & 15.9 \\
\hline 5 & 16.4 \\
\hline
\end{tabular}

angle absorption due to emission originating from the point $\left(r^{\prime}, \varphi^{\prime}\right)$ is approximately equal to the total absorption in the point $\left(r=r^{\prime}, \varphi=\varphi^{\prime}\right)$ due to cone angle emission from neighboring points in the LSC plain. The cone angle correction factor for the absorption $\gamma_{c a, i}(r, \varphi, \lambda)$ is therefore given by Eq. (16). This correction factor can now be added to the original absorption $\gamma_{i}$ [Eq. (14)] and be used to calculate a new emission and a new edge transmission.

The results for a $3 \mathrm{D}$ circular LSC with a radius of $0.5 \mathrm{~m}$ and a $\eta_{\mathrm{LQE}}$ of $80 \%$ are shown in Table 3 . Without taking into account the cone angle absorption the transmitted fraction would be $14.6 \%$ of the initially absorbed photons. This number increases to $14.7 \%$ for an LSC with a thickness of $0.1 \mathrm{~mm}$ and to $16.4 \%$ for an LSC thickness of $5 \mathrm{~mm}$. So, for relatively thick LSCs this contribution should be included in the calculations. Note that, although Table 3 seems to suggest that the self-absorption losses can be reduced by using thicker LSCs, this is only partly true. In a thicker LSC the cone angle losses in each generation of emission are reduced. However, if the $\eta_{\mathrm{LQE}}$ is not $100 \%$, each reemission step is still associated with losses due to a nonunity $\eta_{\mathrm{LQE}}$. Furthermore, the transmitted fractions shown in Table 3 are still far from the $59.6 \%$ that would be achieve $\bar{d}$ when there is no self-absorption.

\section{Conclusions}

An analytical model has been presented with which the effects of self-absorption in a luminescent solar concentrator (LSC) can be described. The equations only require the absorption and emission spectra, the quantum efficiency of the concerning dye, the LSC dimensions, and the refractive index as input parameters, and they can be solved easily using standard commercial math software, as opposed to more elaborate ways of modeling self-absorption such as Monte Carlo simulations. In this work, the model was used to describe self-absorption by a Red 305 dye as an example, but the model is applicable to any other luminescent material for which the absorption and emission spectrum are known, such as type I and type II quantum dots. By doing this, insight is obtained not only on the efficiency losses due to self-absorption but also on the absorption and emission distribution of the light within the LSC as a function of LSC size and thickness and luminescence quantum efficiency. The calculations may furthermore be extended with equations describing other losses within a LSC, such as those associated with light scattering.

This work was part of the Joint Solar Programme (JSP) of the Stichting voor Fundamenteel Onderzoek der Materie (FOM) and financially supported by HyET Solar.

\section{References}

1. M. G. Debije and P. C. Verbunt, "Thirty years of luminescent solar concentrator research: solar energy for the built environment," Adv. Energy Mat. 2, 12-35 (2012).

2. B. C. Rowan, L. R. Wilson, and B. S. Richards, "Advanced material concepts for luminescent solar concentrators," IEEE J. Sel. Top. Quantum Electron. 14, 1312-1322 (2008).

3. W. G. J. H. M. van Sark, K. W. J. Barnham, L. H. Slooff, A. J. Chatten, A. Büchtemann, A. Meyer, S. J. McCormack, R. Koole, D. J. Farrell, R. H. Bose, E. E. Bende, A. R. Burgers, T. Budel, J. Quilitz, M. Kennedy, T. Meyer, C. De Mello Donegá, A. Meijerink, and D. Vanmaekelbergh, "Luminescent solar concentrators: a review of recent results," Opt. Express 16 21773-21792 (2008).

4. T. Dienel, C. Bauer, I. Dolamic, and D. Brühwiler, "Spectralbased analysis of thin film luminescent solar concentrators," Sol. Energy 84, 1366-1369 (2010).

5. L. R. Wilson and B. S. Richards, "Measurement method for photoluminescent quantum yields of fluorescent organic dyes in polymethyl methacrylate for luminescent solar concentrators," Appl. Opt. 48, 212-220 (2009).

6. A. Goetzberger and W. Greubel, "Solar energy conversion with fluorescent collectors," Appl. Phys. 14, 123-139 (1977).

7. A. A. Earp, G. B. Smith, P. D. Swift, and J. Franklin, "Maximizing the light output of a luminescent solar concentrator," Sol. Energy 76, 655-667 (2004).

8. J. W. E. Wiegman and E. van der Kolk, "Building integrated thin film luminescent solar concentrators: detailed efficiency characterization and light transport modelling," Sol. Energy Mater. Sol. Cells 103, 41-47 (2012).

9. J. Sansregret, J. M. Drake, W. R. L. Thomas, and M. L. Lesiecki, "Light transport in planar luminescent solar concentrators: the role of DCM self-absorption," Appl. Opt. 22, 573-577 (1983)

10. T. Wang, B. Yu, B. Chen, Z. Hu, Y. Luo, G. Zou, and Q. Zhang, "A theoretical model of a cylindrical luminescent solar concentrator with a dye-doping coating," J. Opt. 15, 055709 (2013).

11. J. S. Batchelder, A. H. Zewail, and T. Cole, "Luminescent solar concentrators. 1: theory of operation and techniques for performance evaluation," Appl. Opt. 18, 3090-3110 (1979).

12. J. S. Batchelder, A. H. Zewail, and T. Cole, "Luminescent solar concentrators. 2: experimental and theoretical analysis of their possible efficiencies," Appl. Opt. 20, 3733-3754 (1981).

13. R. W. Olson, R. F. Loring, and M. D. Fayer, "Luminescent solar concentrators and the reabsorption problem," Appl. Opt. 20, 2934-2940 (1981).

14. V. Sholin, J. D. Olson, and S. A. Carter, "Semiconducting polymers and quantum dots in luminescent solar concentrators for solar energy harvesting," J. Appl. Phys. 101, 123114 (2007).

15. D. Sahin and B. Ilan, "Monte-Carlo simulations of light propagation in luminescent solar concentrators based on semiconductor nanoparticles," J. Appl. Phys. 110, 033108 (2011).

16. A. Kerrouche, D. A. Hardy, D. Ross, and B. S. Richards, "Luminescent solar concentrators: from experimental validation of $3 \mathrm{D}$ ray-tracing simulations to coloured stained-glass windows for BIPV," Sol. Energy Mater. Sol. Cells 122, 99-106 (2014). 
17. A. J. Chatten, K. W. J. Barnham, B. F. Buxton, N. J. EkinsDaukes, and M. A. Malik, "A new approach to modelling quantum dot concentrators," Sol. Energy Mater. Sol. Cells 75, 363-371 (2003).

18. L. Fang, T. S. Parel, L. Danos, and T. Markvart, "Photon reabsorption in fluorescent solar collectors," J. Appl. Phys. 111, 076104 (2012).
19. D. Sahin and B. Ilan, "Radiative transport theory for light propagation in luminescent media," J. Opt. Soc. Am. A 30, 813-820 (2013).

20. L. Desmet, A. J. M. Ras, D. K. G. de Boer, and M. G. Debije, "Monocrystalline silicon photovoltaic luminescent solar concentrator with $4.2 \%$ power conversion efficiency," Opt. Lett. 37, 3087-3089 (2012). 